\title{
A multidimensional approach to the generation of helmets' design criteria: a preliminar study
}

\author{
Alemany, $\mathrm{S}^{\mathrm{a}}$; Olaso, $\mathrm{J}^{\mathrm{a}}$; Nacher, $\mathrm{B}^{\mathrm{a}}$; Gil, $\mathrm{M}^{\mathrm{a}}$; Hernández, $\mathrm{A}^{\mathrm{a}}$; Pizá, $\mathrm{M}^{\mathrm{a}}$; Solves, $\mathrm{C}^{\mathrm{a}}$ \\ ${ }^{a}$ Instituto de Biomecánica de Valencia. Universidad Politécnica de Valencia. Edificio 9C. Camino de Vera $s / n$ E- \\ 46022. Valencia, Spain
}

\begin{abstract}
The design and development process of helmets incorporates systematically design criteria related to safety to accomplish European and local standards for the commercialization. However, there are few studies focused on user's comfort and adaptation. Present study tackles a multidimensional approach to gain better understanding of the interaction between helmet and user to generate design criteria for the internal helmet surface. Morphological characteristics of the target population, pressure distribution over head and subjective perception of fitting and discomfort are the factors considered to establish the criteria that assure a proper fit. Ten men corresponding to helmet size $\mathrm{M}$ and usual drivers of motorbike wore two helmet models in three sizes (S, M and L). The head shape of participants was acquired using the head scanner of I-Ware laboratory and an instrumented pad was used to measure pressure in five head regions. After wearing the helmet, users filled in a perception questionnaire about fitting, comfort and usability considering the five regions. Users' fitting perception provided the relation between pressure levels and the comfort felt in the five regions. This study constitutes a first approach to a new methodology to generate criteria to improve the design of helmets under a multidimensional approach.
\end{abstract}

Keywords: Anthropometry, head scanning, fitting, pressure, helmet

\footnotetext{
* Corresponding author. Tel: +34 9638791 60; fax: +34963879169
}

E-mail address: Sandra.alemany@ibv.upv.es 


\section{Introduction}

Helmet is essential protective equipment to injury prevention in case of accidents in sport, transport and working activities. For each application, there is much information regarding injury incidence and the more frequent areas of impact on the head as well as specific regulations checked with compulsory certifications. In contrast, there is a lack of information about design criteria addressing the achievement of a suitable fitting of helmets. Correct fitting is important for comfort but also to optimize protection. Tight fitting generates excessive compression of inner materials and, in consequence, a dramatic reduction of the shock absorption capacity. On the contrary, a loosed helmet does not correctly match head's morphology and may move, which have negative effects: reducing shock absorption capacity, increasing inertia and vibrations, and also can block driver's field of view. In addition, on both cases there is a high risk that the user leaves the use of helmet due to discomfort.

The emergence of 3D head scanners providing detailed information of the head surface motivated early investigations about helmet fitting. These studies have been focused on the improvement of anthropometric methods and the aligning procedure to functionally match head al helmet geometry [7], [8]. A fitting improvement of helmets was firstly achieved by the sizing optimization. Meunier et al. [4] used de standoff distance (distance between the inside of the helmet and the skull) for the assessment of helmet fit and suggested a helmet sizing criteria derived from this parameter to better accommodate a given population. Harrison and Robinette [2] developed a scientific method based on fit testing on living subject for determining the minimum number of aviator helmet sizes to accommodate the full anthropometric variability of the military aviator population. However, as shown in this [7] and other research fields [9], anthropometry is not enough to achieve good fitting. In order to determine useful helmet design parameters to assure correct fitting from the comfort and protection point of view, the physical interaction between helmet and head should be also analyzed considering geometry but also material deformation. Helmet-head pressure distribution has been studied using an array of 13 flexible sensors during an impact simulation done with a drop test [5], [6]. But related to comfort, it was Chung Hee [3] who used pressure sensors to study comfort perception of baseball caps. A relationship between pressure and subjective perception was obtained by regression equations, proven the feasibility to analyze fitting measuring interaction pressures. A device based on single point sensors was developed to evaluate the pressure on eight points of two mannequin heads representing two different head sizes. However, two mannequins' heads does not represent the effect of 3D shape variability of human heads.

The aim of this research is to set up a methodology to generate design criteria for optimum fitting of helmets based on a multidimensional approach [1] relating geometry (anthropometry of the head and inner shape of the helmet), pressure interaction and subjective perception.

\section{Methods}

Ten men corresponding to helmet size $\mathrm{M}$, aged 2050 years, without pathologies and usual drivers of motorbike wore two models of helmet in three sizes (Small, Medium and Large). The models were a closed sportive helmet and an executive helmet with a front opening system (figure 1).

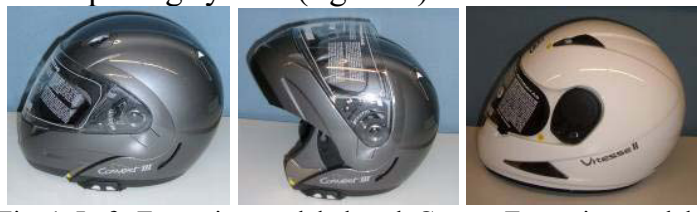

Fig. 1. Left: Executive model closed. Centre: Executive model closed. Right: Sportive model.

\subsection{Morphologic head characterization}

The head shape of participants was acquired using the head scanner of I-Ware laboratory, an optical laser scanning system formed by 6 laser beams and 12 cameras mounted on a circular framework.

A white head cap made of elastic textile was worn by the subject (figure 2) to avoid hair irregularities on the scanned geometry. The subjects sat down upright looking at front and the circular framework was moved from the top of the head to the neck in 10 seconds to perform the $3 \mathrm{D}$ registration of the head (figure 3).

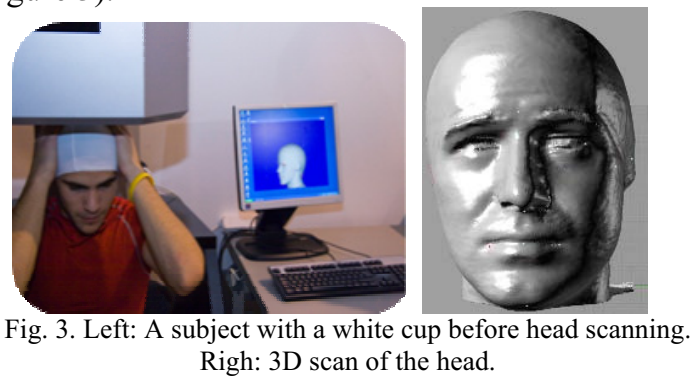

Cranial surface was divided into five different regions (frontal, back, top, left side and right side) and 
a set of 25 anthropometric measures (figure 4) were semi-automatically obtained based on 22 anatomical landmarks using a specifically plug-in developed with the CAD tool for 3D modeling Rhinoceros 4.0.
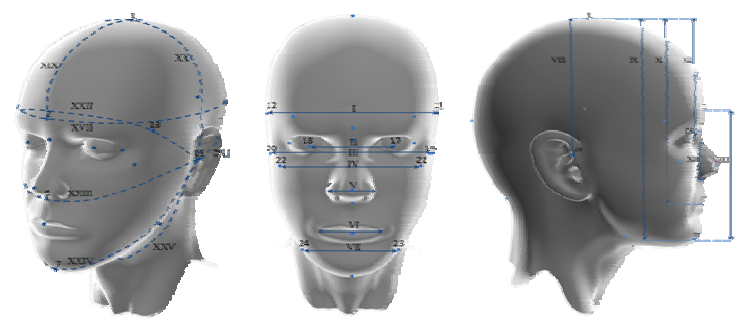

Fig. 4. Anatomical landmarks and anthropometric measures calculated from the $3 \mathrm{D}$ scan of the head.

\section{2. $3 D$ model of the helmet}

In order to analyze geometric interaction between head and helmet the inner surface of the helmet was digitized and aligned with the user's head. The reconstruction of the 3D CAD model of the helmet was based on critical sections digitalized with the contact arm Microscribe-3DX with an accuracy of $0.21 \mathrm{~mm}$ (figure 5).
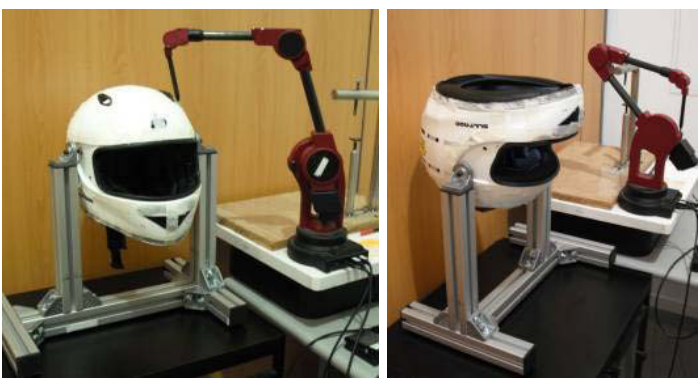

Fig. 5. Left: Digitalization of the outer shape of the helmet. Right: Digitalization of the inner shape of the helmet.

A customized structure was build to fix the helmet enabling to rotate it around two points and digitalize the inner shape controlling the position. The inner shape was modeled over the polystyrene element taking out the soft pads (figure 5).

Inner elements and pads were modeled according to the geometric dimensions provided manufacturing by the company using Rhinoceros 4.0 (figure 6). Six CAD models were obtained representing the two models on the three sizes.

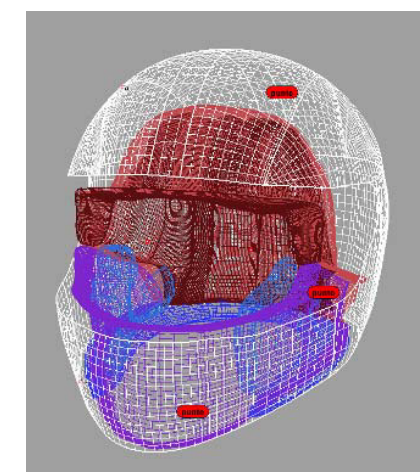

Fig. 6. 3D CAD model of the helmet.

\subsection{Head and helmet alignment}

Motorbike helmets sit different for each uses depending on the shape of the users' head, the deformation of the inner soft pads of the helmet and the peripheral elements such us optics or ear cups [8].

The alignment of the 3D head and helmets were done following the approach proposed by Whitestone and Robinette [8] for aviator helmets. This approach used a scan of the user wearing the helmet as the reference to match the single $3 \mathrm{D}$ head scan and the $3 \mathrm{D}$ model of the helmet.

The scan of the user wearing the helmet (figure 7) was done with the laser body scanner Vitus Smart XXL from Human Solutions with a density of points of 27 points $/ \mathrm{cm}^{2}$ and an average maximum error on circumference lower than $1 \mathrm{~mm}$.

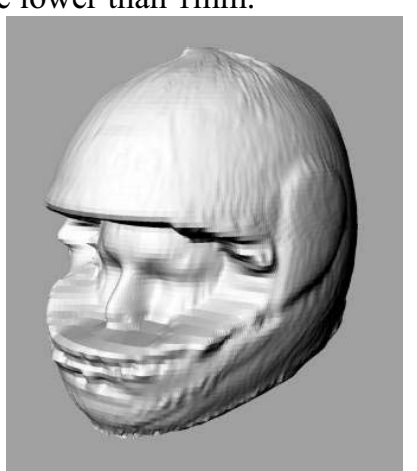

Fig. 7. 3D scan of the head of the user wearing the helmet.

Four reference points marked and rigidly attached to the external surface of the helmet were used to perform the registration of the $3 \mathrm{D}$ CAD model of the helmet and the scan of the subject wearing the helmet. These points were digitalized on both acquisition process described before.

The registration of the single scan of the head and the scan of the subject wearing the helmet was based on three common anatomical landmarks: left and right ectocanthion (most external point of the orbital) and pronasale (most prominent point of the nose). 
These landmarks were selected due to a good accessibility for their registration when the user wears the helmet and because they obtain the best intraclass correlation coefficient in a repeatability study.

The registration processes were done minimizing the Euclidian distance between homologous landmarks (figure 8).
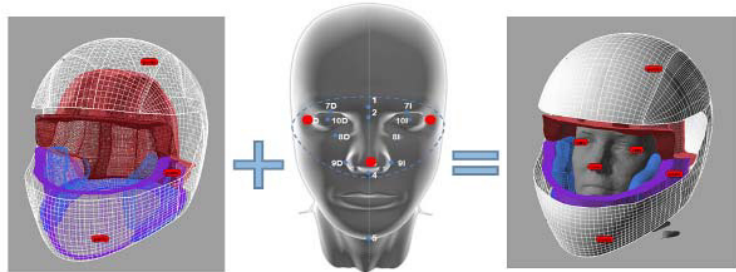

Fig. 8. Registration process to align the CAD model of the helmet with the head scan.

Interference areas and volumes between the inner surface of the helmet and head surface were calculated to analyze geometric fitting (figure 9).

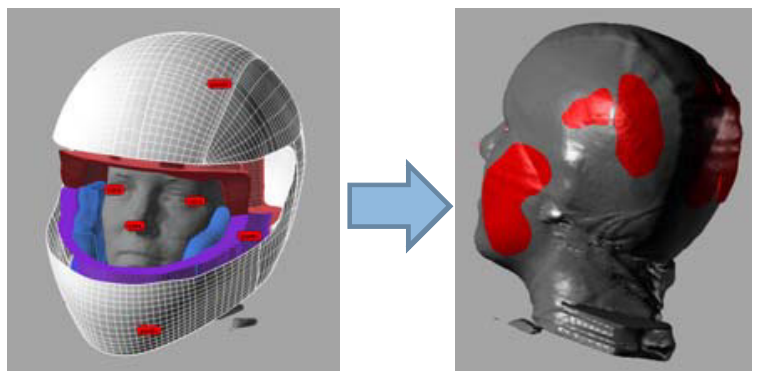

Fig. 9. Calculation of interference areas and volumes between the head shape and the inner surface of the helmet.

\subsection{Pressure distribution}

An stretchable array instrumented with a $16 \times 16$ pressure sensors with a resolution of $1 \mathrm{~cm}$ and active area of 160x160 mm form Pressure Profile Systems was used to measure pressure distribution between head and helmet.

The head was divided in 5 regions in order to map the pressure of the whole head (figure 10). The pressure pad was hold with a textile cup enabling their placement and avoiding their movement when the user put on the helmet. A cero set up was done after the pad is fixed on the head in order to remove the pressure provided by the textile cup and the pressure due to the deformation of the pad to be adapted to head curvature.
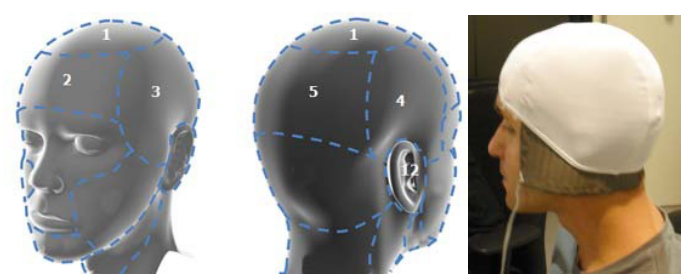

Fig. 10. Left: Regions of the head measured with the pressure pad. Right: Location of the pressure pad by using a elastic textile cup.

The visualization software Chamaleon TVR version 1.3.9.2. (Figure 11) was used to perform the acquisition and visualization of the pressure maps. Pressure measures of the 16 sensors were registered during 10 seconds.

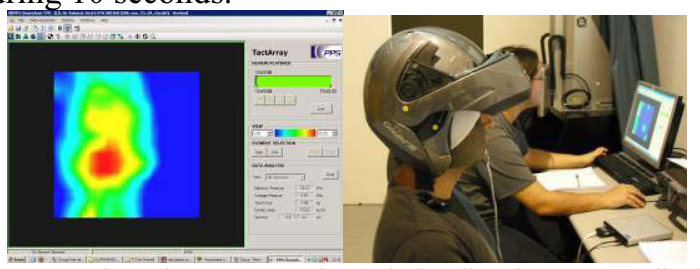

Fig. 11. Left: Software to acquire and visualize the pressure distribution. Right: Instrumentation of the user with the pressure pad.

From pressure data it was calculated:

- The peak pressures measured by each sensor (DMMAX).

- The maximum pressure of the DMMAX.

- The mean pressure of the DMMAX.

\subsection{Subjective assessment}

The 10 subjects tried on the 3 sizes of the two helmets. When a helmet was too small that it was impossible to be put on (usually on size S) the trial was neglected. While wearing the helmet, users answered a questionnaire about fitting perception and preference focusing the five cranial regions. The global comfort perception was also rated by the subjects in a scale ranging from 1 to 7 (table 1).

$$
\text { Table } 1
$$

Rating scale to assess subjective comfort.

\begin{tabular}{|c|c|}
\hline Comfort perception & Rate \\
\hline Very uncomfortable & 1 \\
\hline Uncomfortable & 2 \\
\hline Slightly uncomfortable & 3 \\
\hline Neutral & 4 \\
\hline Slightly comfortable & 5 \\
\hline Comfortable & 6 \\
\hline Very comfortable & 7 \\
\hline
\end{tabular}

Statistical treatment was applied to measured data with SPSS 16 software. ANOVAS and correlations were calculated to extract the relations between the different factors considered: user's anthropometry and fitting perception, helmet type, size and mor- 
phology, and pressure applied by the helmet on the head.

\section{Results}

\subsection{Effect of the helmet model in the pressure patterns}

Closed helmet significantly increased mediolateral pressures (sum up of peak pressures on regions 3 and 4) compared to open helmet models (table 2). The mean pressure difference on this axis was $3.89 \mathrm{~Pa}$ (figure 12).

Table 2

Significance of the ANOVA test comparing pressure provide by each model of helmet.

\begin{tabular}{|c|c|}
\hline Pressure axis & ANOVA Significance \\
\hline Medio-lateral (Regions 3 and 4) & 0.014 \\
\hline Antero-posterior (Regions 2 and 5) & 0.293 \\
\hline
\end{tabular}

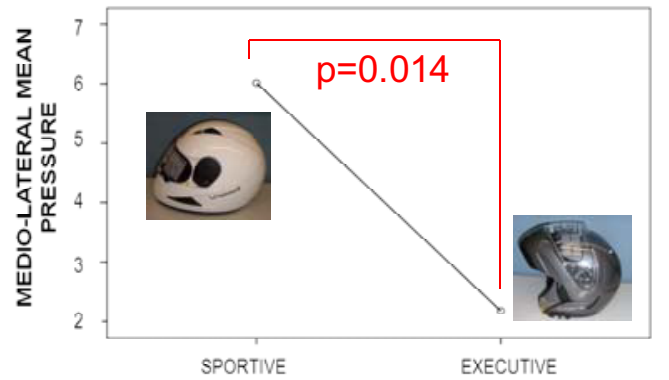

Fig. 12. Mean pressure provided by the sportive and executive helmet models on regions 3 and 4 .

The figure 13 shows the pressure distribution on region 3 of one of the subjects for the sportive and executive helmets. The left side with higher pressures corresponds to the pressure near the cheek and the right side corresponds to the pressure near the ear. While both helmets shoe a similar pressure pattern (more pressure on the cheek than in the area near the ear) the sportive helmet generates higher pressures on the area of the cheek.

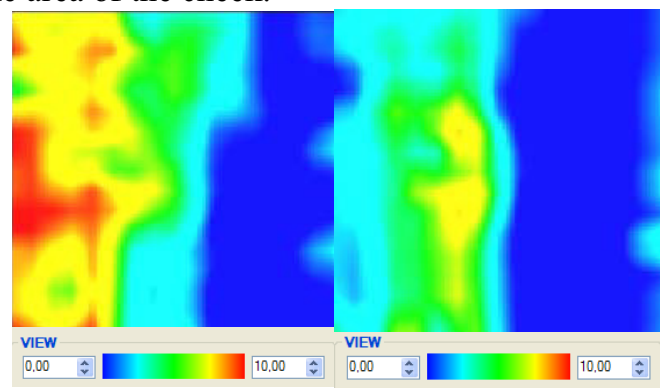

Fig. 13.Left: Pressure distribution $(\mathrm{Pa})$ provided by model sportive size $\mathrm{L}$ on region 3 for subject 08 ; Right: Pressure distribution $(\mathrm{Pa})$ provided by model executive size $\mathrm{L}$ on region 3 for subject 08 .
Any significant difference was found on anteroposterior axis (sum up of peak pressures on regions 2 and 5) between the helmet models. The mean pressure on this axis considering both models is $2.86 \mathrm{~Pa}$ (figure 14).

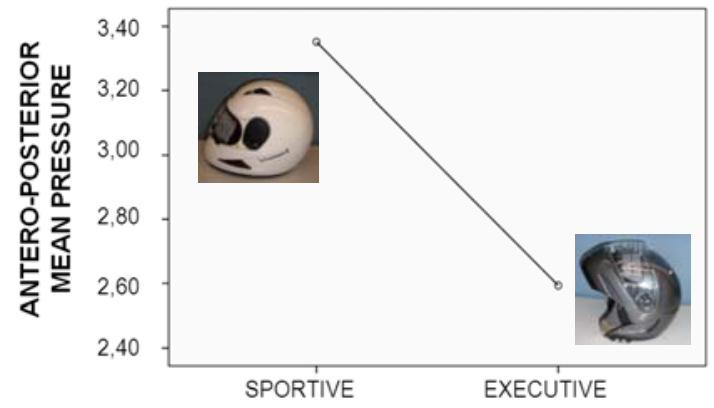

Fig. 14. Mean pressure provided by the sportive and executive helmet models on regions 2 and 5 .

\subsection{Effect of the size in the pressure patterns}

The ten subjects participating on the study usually worn helmet size M. Pressure provided by helmets sized $\mathrm{S}$ were expected to be higher while pressure provide by helmet sized L were expected to be lower.

Results confirmed this fact for the sportive model, providing significant statistical differences between size $\mathrm{S}$ and $\mathrm{L}$ (figure 15) on the antero-posterior axis (sum up of peak pressures on regions 2 and 5).

On the other hand, pressure on the medio-lateral axis showed higher intra-size variability and significant differences have not been obtained (table 3 ). Mean pressure values are similar to that measured on the antero-posterior axis (figure 16).

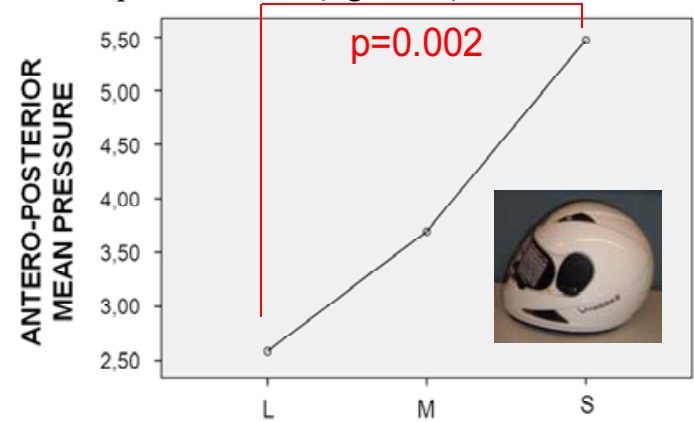

Fig. 15 Mean pressure provided by sportive helmet for each size on regions 2 and 5.

Table 3.

Significance of the ANOVA test comparing pressure provided by sportive helmet model on sizes $\mathrm{S}, \mathrm{M}, \mathrm{L}$.

\begin{tabular}{|c|c|}
\hline Pressure axis & ANOVA Significance \\
\hline Medio-lateral (Regions 3 and 4) & 0.216 \\
\hline Antero-posterior (Regions 2 and 5) & 0.002
\end{tabular}




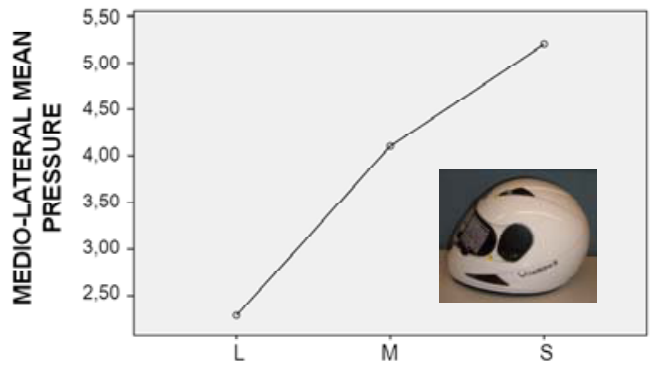

Fig. 16.Mean pressure provided by sportive helmet for each size on regions 3 and 4.

\subsection{Influence of head anthropometry on pressure pattern}

Only significant correlations between anthropometry and pressure on the frontal region (region 2) were obtained on size $\mathrm{M}$, which is the preferred size selected by all the subjects (table 4). Longer head breadth, length and perimeter (figure 17) entailed higher pressures in the frontal region.

\section{Table 4}

Significance of the ANOVA test comparing pressure on region 2 provided by both type of helmets on sizes $\mathrm{M}$.

\begin{tabular}{|c|c|}
\hline Anthropometry of the head & ANOVA Significance \\
\hline Breadth & 0.629 \\
\hline Length & 0.792 \\
\hline Perimeter & 0.611 \\
\hline
\end{tabular}
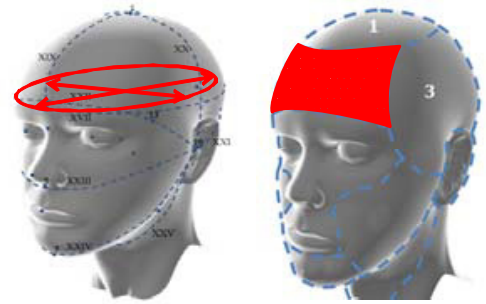

Fig. 17 Left Representation of anthropometric measures: breadth, length and perimeter. Right: Location of pressure pad on region 2.

\subsection{Relationship between pressure patterns and users' perception}

Significant correlations between pressure and user's perception of fitting comfort were obtained on the antero-posterior axis, medio-lateral axis and cheeks (figure 18): Lower pressures on these areas entailed lower level of comfort perceived (table 5).

$$
\text { Table }
$$

Correlation coefficients and level of significance between pressure in different areas and perceived comfort.

\begin{tabular}{|l|c|c|}
\hline Area & Correlation & Significance \\
\hline Antero-Posterior cranium & 0.423 & 0.022 \\
\hline Medio-lateral cranium & 0.416 & 0.025 \\
\hline Cheeks & 0.523 & 0.004 \\
\hline
\end{tabular}

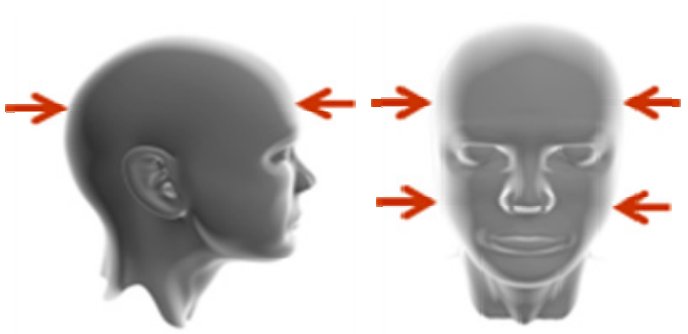

Fig. 18. Antero-Posterior, medio-lateral and cheeks axis.

These results (an increase on pressure is related to an increase to fitting comfort) suggest that once you can wear a helmet you like it tightly attached.

\subsection{Relationship between head anthropometry and the geometric interference}

The areas and volumes of the geometrical interference calculated from the virtual alignment of the $3 \mathrm{D}$ head scan and the 3D CAD model of the helmet were correlated with anthropometric measures of the head to analyze their consistency.

Significant correlations were obtained between the volume of the interference in the top head area (region 1) and head breath, head length and head circumference for the executive helmet model (table 6) and head length and bitragion arch (figure 19) for the sportive helmet model (table 7).

$$
\text { Table } 6
$$

Correlation coefficients and level of significance between the volume of interference and anthropometric measures of the head for the executive helmet model.

\begin{tabular}{|l|c|c|}
\hline Area & Correlation & Significance \\
\hline Head breadth & 0.613 & 0.045 \\
\hline Head length & 0.617 & 0.043 \\
\hline Head circumference & 0.677 & 0.022 \\
\hline
\end{tabular}

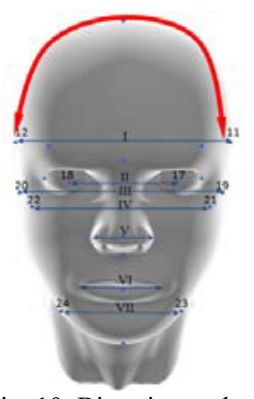

Fig. 19. Bitragion arch

Table 7

Correlation coefficients and level of significance between the volume of interference and anthropometric measures of the head for the sportive helmet model.

\begin{tabular}{|l|c|c|}
\hline Area & Correlation & Significance \\
\hline Head length & -0.835 & 0.043 \\
\hline Bitragion arch & 0.833 & 0.022 \\
\hline
\end{tabular}


A negative correlation was obtained between the head length and the volume of interference for the sportive helmet. This could be explained by a lack of contact in the top of the head for longer heads, resulting on a different accommodation pattern compared with the executive model.

\section{Conclusions}

A new multidimensional methodology was developed and applied to investigate the ergonomy of motorbike helmets.

The analysis of pressure distribution maps to quantify fitting interaction between head and helmet has proved to be related to user's fitting perception. In addition, geometric interferences between helmet and head morphologies have resulted to be correlated to head anthropometry.

These results open the possibility to work on simulation tools supporting the design process predicting pressure distribution on the head surface and relating it with subjective perception.

These promising results are the first approach to a future deeper research on each aspect of this methodology to obtain concrete models enabling the prediction of user comfort based on geometry and pressure interaction.

\section{Acknowledgments}

This project has been partially funded by the MEC (reference DPI2007-66245-C02-02). The authors of this paper would like to thank the company NZi who provides the sample of helmets.

\section{References}

[1] S. Alemany, J. C. González, A. C. García, J. Olaso, J. Montero, C. Ghirivella, J. Prat, and J. Sánchez, "A novel approach to define customized functional design solution from user information," the 3rd Interdisciplinary World Congress on Mass Customization and Personalization, Hong Kong, CD-ROM, 2005

[2] C.A. Harrison and K.M. Robinette K.M. Principles of fit to optimize helmet sizing, Proceedings of the NATO Research Technology Organization's Human Factors \& Medicine Panel Symposium on Strategies to Maintain Combat Readiness During Extended Deployments: A Human Systems Approach, Prague, Czech Republic, 3-5 October, 2005, pp.

[3] C. Hee Park, Y. Jun, T. Jin Kang and J. Hyun Kim Development of a Tool to Measure the Pressure Comfort of a Cap (II) by the Analysis of Correlation Between Objective Pressure and Subjective Wearing Sensation. Textile Research Journal 2007; 77,n.7, p.p. $520-527$

[4] P. Meunier, D. Tack, A. Ricci, L. Bossi and H. Angel, Helmet accommodation analysis using 3D laser scanning. Applied Ergonomics 31 (2000) 361-369.

[5] R. Ouckama and Pearsall DJ (2008) Dynamic pressure mapping of the head-helmet interface. North American Congress of Biomechanics (NACOB), Ann Arbor, Michigan, 5-9 August 2008

[6] R. Ouckama and David J. Pearsall. Evaluation of a flexible force sensor for measurement of helmet foam impact performance. Journal of Biomechanics 44 (2011) 904-909.

[7] K. M. Robinette and J. J. Whiteston. The need for improved anthropometric methods for the development of helmet systems. Aviat. Space Environ. Med. 1994; 65(4, Suppl.): A9599, Aerospace Medical Association, Alexandria, VA.

[8] J. J.Whiteston, Design and evaluation of helmet system using 3D data. Proceedings of the Human Factors and Ergonomics Society 37 th annual meeting, 1993, pp. 64-68.

[9] J. Olaso, J.C. González, S. Alemany, E. Medina, A. López, C. Martín, J. Prat, C. Soler. Study of the influence of fitting and walking condition in foot dorsal pressure. Proceedings of the VIII $^{\mathrm{TH}}$ footwear biomechanics symposium. Edited by E.D. Frederick \& S.W. Yang. National Yang-Ming University. Taipei, Taiwan. June 27-29, 2007. 